\title{
Transmission Efficiency Analysis and Experiment Research of Gear Box Jing $\mathrm{LI}^{1}$, Chang-Chun $\mathrm{LI}^{1, \mathrm{a}}$ and Jing HUANG ${ }^{1}$ \\ 1School of Mechanical, Electronic and Control Engineering, Beijing Jiaotong University, Beijing 100044, PR China
}

Keywords: Transmission efficiency, Gear box, Energy storage device.

\begin{abstract}
Transmission efficiency is a key characteristic for gear box, a test system is set up for gear box's measurement. But in the test process, there may have some wrong test results by using ideal mathematic formula which is based on the law of conservation of energy. By checking the mechanical structure of system and analyzing the experiment data, there are some interference factors in this test system. The main interference factor is that there exist some energy storage devices in this test system, when the system is instead state these energy storage devices also can supply some energy to output. If this part of energy is not considered in the mathematic calculation, the test result is wrong which means the transmission efficiency may over than $100 \%$. In order to get the correct transmission efficiency, a new calculation method is used to eliminate the interference factors from the mathematical calculation. With this new method, the experiment process is carried out repeatedly to achieve a decent test result, which illustrates the correctness of the analysis and mathematical calculations.
\end{abstract}

\section{Introduction}

The transmission gear box or reduction gear box is used wildly in many fields like aerospace field and automotive field [12]. The transmission efficiency is a key characteristic for gear box, because gears are used to transmit motion and/or power [3]. Many researchers already have done some research works of gear box's transmission efficiency [4]-[9]. In order to research the transmission efficiency of gear box, we build a test measurement system. But in some special cases, the test result maybe is wrong if followed the traditional calculation method, because the effect of interference factors [10]. So the interference factors must be found out and eliminated from the mathematical calculations.

\section{The Measurement Method}

The test system's mechanical structure is shown in Figure1, this system mainly consists of three parts: X axis active load (I), Y axis active load (II) and the drive part (III). These three parts use servo motorsas the power supply, and use torque sensors (10, 4 in Figure 1) to test the input and output's torque and speed.

The traditional method of transmission efficiency measurement is realized by calculating withthe rotation rate and torque of the system's input and output. So there installs a rotation rate and torque sensor at the system's input, and installs another one at the system's output. The system's transmission efficiency $E_{t}$ can be calculated with Eq. (1). 


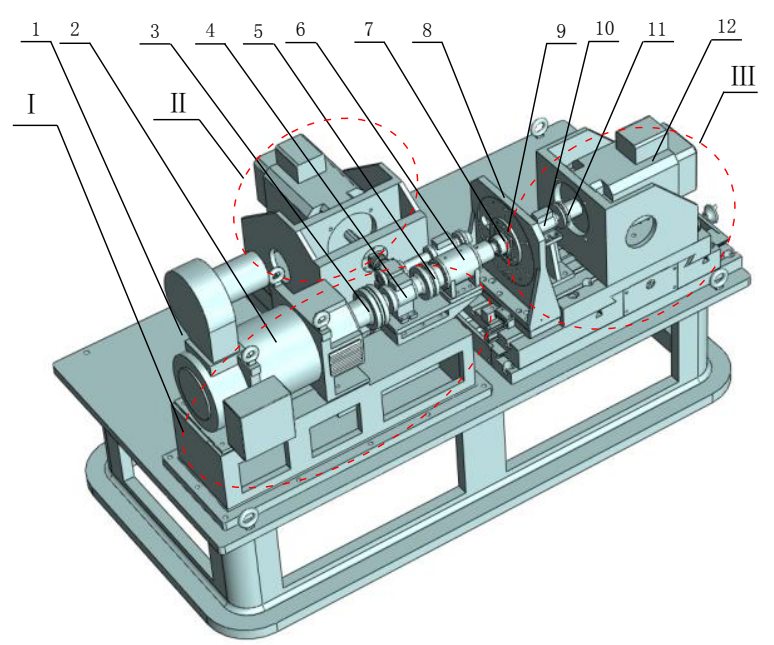

Figure1. Mechanical Structurediagram

1- base. 2- loading motor. 3,5,11- membrane coupling.

4,10- torque speed sensor. 6- bearing support. 7- gear box

8- gear box support. 9- mechanical chuck. 12- drive motor

$$
E_{t}=\frac{S_{0} T_{0}}{S_{i} T_{i}}
$$

where $S_{0}, S_{i}$ are the rotation rate of system's output and input, respectively. ${ }^{T_{0}, T_{i}}$ are the torque of system's output and input, respectively.

This is an ideal calculate method of transmission efficiency. But when we used this calculate method in our system, one problem is that the transmission efficiency value often exceeds $100 \%$. The calculate result is shown in Figure 2.

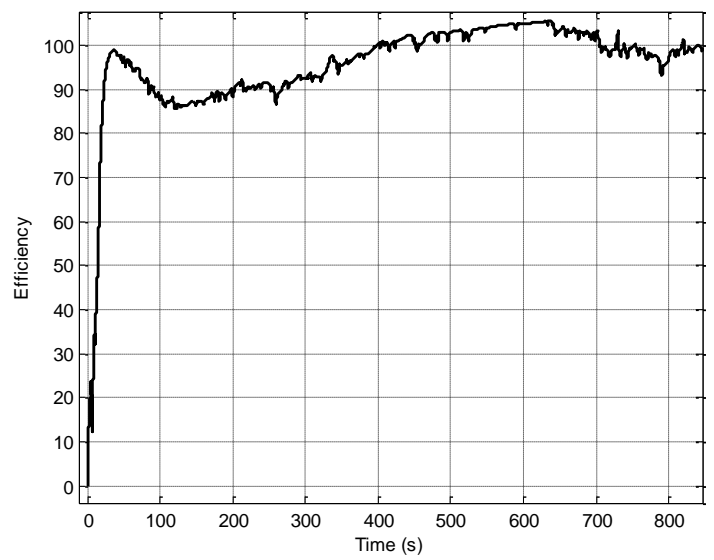

Figure2. Transmission efficiency

Obviously, the measurement result is wrong, because the transmission efficiency is always less than $100 \%$. So there must be some interference factors we do not take into account when we use the Eq.(1).

If check the system's input and output torque which is shown in Figure 3, and check the system's input and output power which is shown in Figure 4,we can find out that the torque and power of input is less than the output, when system's output torque reached the setting value $(5 \mathrm{Nm})$ after 400 seconds, until the measurement was finished after 700 seconds.

According to the law of conservation of energy, the total input energy is equal to the total output energy plus total consumption energy. If the input energy is less than output, there must be another energy supply point between input measurement point and output measurement point. 


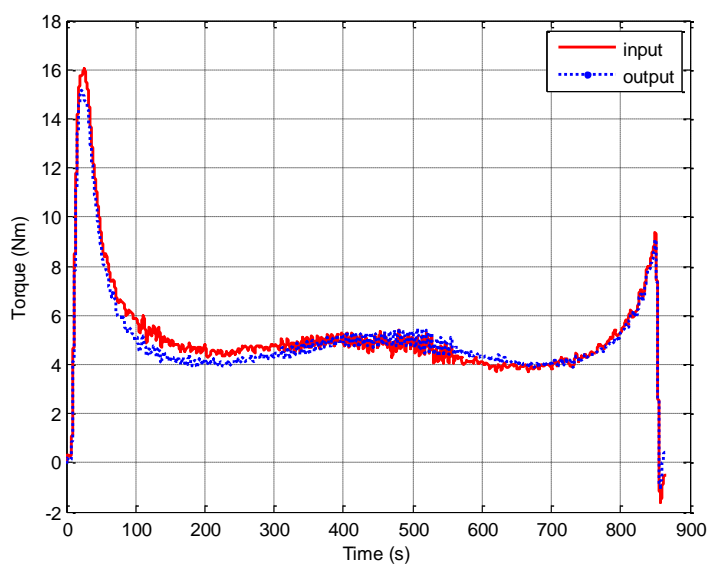

Figure3. Input and output torque

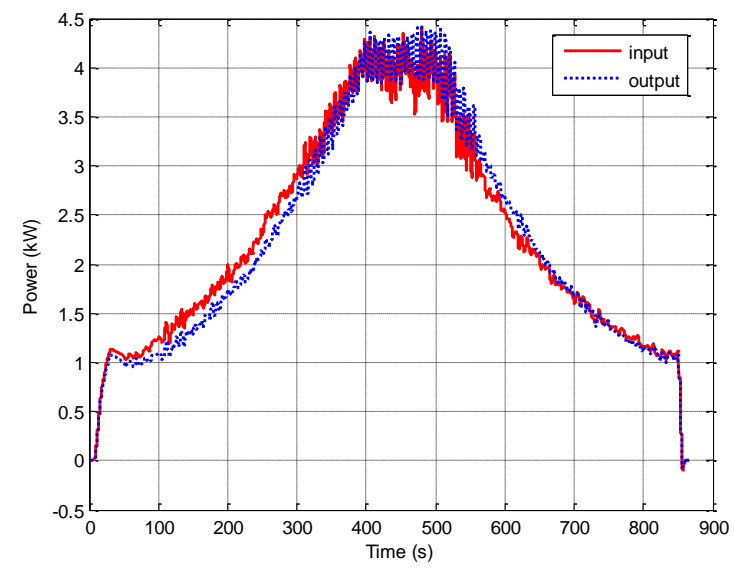

Figure4. Input and output power

In the real world, there are always some interference factors like energy storage devices in this system. If there are flexible transmission shafts, some elastic components and heavy moment of inertia components in a system, these kinds of components will store the energy when test the transmission efficiency and affect the measurement result.

The coupling of this system is flexible, so it can store some energy by deforming at the beginning of measurement. In this stage, all the energy is supplied by the input of system. When the system is in steady state stage, this coupling component can supply some energy as energy buffer to the output. So in steady state, the system's energy is provided by the input and energy storage devices. Therefore, if we only measure the system's input power and ignore the energy storage devices' influence, we will get the wrong result. The change of system's energy flow can be illustrated by Figure5.

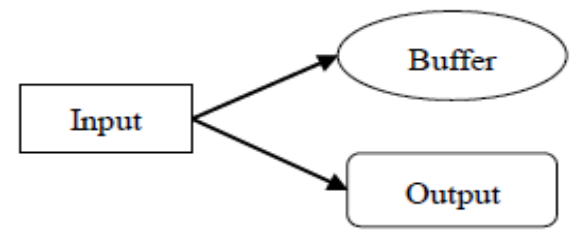

(a)

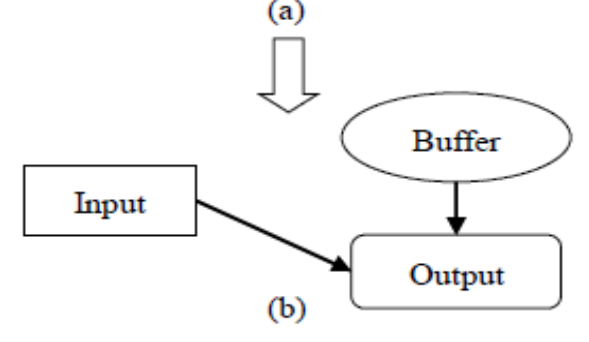

Figure5. The change of system's energy flow

Consider the influence of energy storage devices, the system's energy in Figure 5(a) and Figure 5(b) can be calculated by:

$$
\begin{aligned}
& P_{s 1}+P_{l 1}+P_{o 1}=P_{i 1} \\
& P_{l 2}+P_{o 2}=P_{i 2}+P_{s 2}
\end{aligned}
$$

where $P_{s 1}$ is the stored energy by the energy storage devices.

$P_{s 2}$ is the released energy from energy storage device.

$P_{l 1}, P_{l 2}$ is the loss energy. 
$P_{i 1}$ is the input energy during start-up phase.

$P_{i 2}$ is the input energy after start-up phase.

$P_{o 1}$ is the output energy during start-up phase.

$P_{o 2}$ is the output energy after start-up phase.

The energy $P$ can be calculated by Eq. (4).

$$
P=\int_{0}^{t} T(t) \cdot V(t) d t
$$

where $T$ is the torque and $V$ is the rotation rate.

When the system starts from stationary state and end atstationary state, the initial and end conditions are zeros. So the storedenergy equals to the released energy, which means $P_{s 1}=P_{s 2}$.According to Eqs.(2) and (3), it is able to obtain:

$$
P_{l 1}+P_{l 2}=\left(P_{i 1}+P_{i 2}\right)-\left(P_{O 1}+P_{02}\right)
$$

The total lost energy can be calculated out by the total input energy and the total output energy.

As known, the lost energy is mostly consumed by the mechanical friction. So the lost energy of this system is related to the rotation rate. The faster the speed, the more energy loss. This relationship can be expressed as Eq.(6).

$$
P_{1}=k \cdot V
$$

where $^{k}$ is the proportional coefficient, $V$ is the rotation rate.

According to Eqs. (4) and (5), the proportional coefficient $k$ can be calculated out.

$$
k=\frac{\left(P_{i 1}+P_{i 2}\right)-\left(P_{o 1}+P_{o 2}\right)}{V}
$$

Some experiments are carried out to get the value of $k$ at some certain rotation rate points, i.e., from 500rpm, 1000rpm,1500rpm,till6000rpm. Then get the curve chart of $k$ between 0-6000rpm by using cubic spline interpolation method, which is shown in Figure 6.

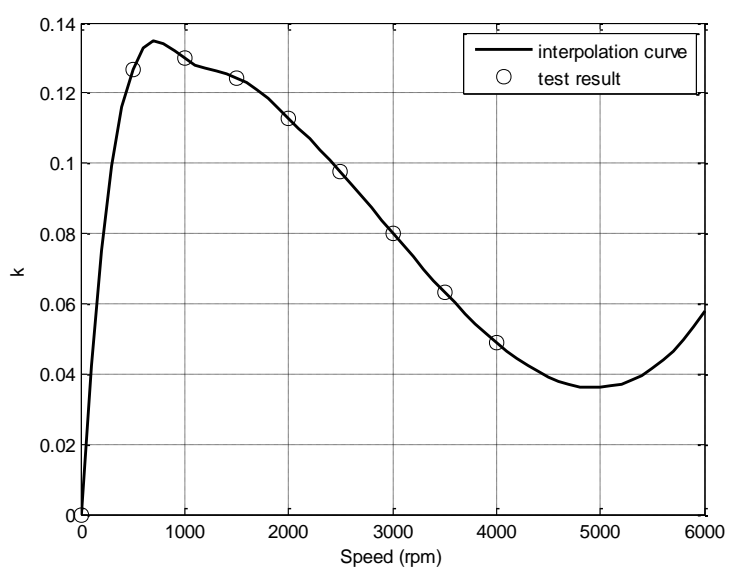

Figure6.Interpolation curve of $k$

The value of $k$ can be obtainedat any rotation rate point from Figure 6 . Then the system's lost energy can be calculated by using Eq.(6), and the system's transmission efficiency ${ }_{t}$ also can be calculated out in real time.

$$
E_{t}=\frac{P_{o}}{P_{l}+P_{o}}=\frac{P_{o}}{k V+P_{o}}
$$




\section{The Actual Application Effect}

After using this new method, the test result never over than $100 \%$ during the whole test process. Take the test result under 5Nm,1000rpm as an example, which is shown in Figure 7.

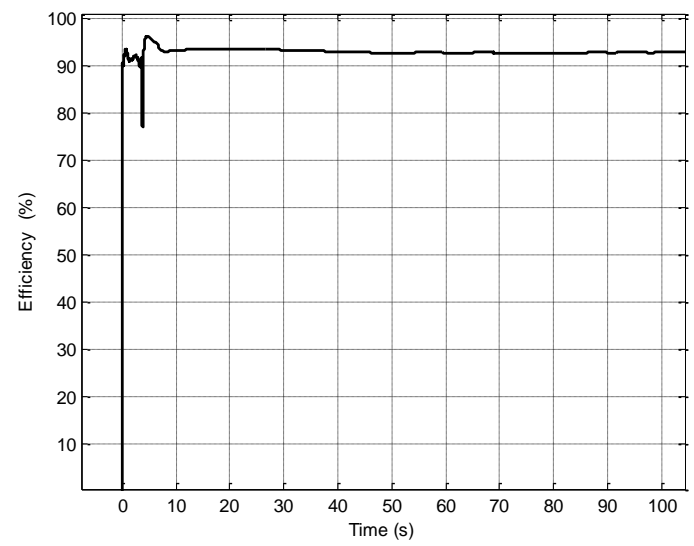

Figure7. New transmission efficiency

The system's transmission efficiency at any speed point can be tested by using this new test method. The test result is shown in Table 1.

Table1. Transmission efficiency at different speed

\begin{tabular}{|c|c|c|}
\hline Torque (Nm) & Speed $(\mathrm{rpm})$ & Average Efficiency \\
\hline 5 & 500 & $86.87 \%$ \\
\hline 5 & 1000 & $89.55 \%$ \\
\hline 5 & 2000 & $87.90 \%$ \\
\hline 5 & 3000 & $88.62 \%$ \\
\hline 5 & 4000 & $93.46 \%$ \\
\hline 35 & 2000 & $97.65 \%$ \\
\hline
\end{tabular}

The test result at the torque $5 \mathrm{Nm}$ can be drawn in a graph. The change trend of transmission efficiency against rotation rate is shown in Figure 8.

From this graph, we can know that the transmission efficiency of this system not always increases with the increase of speed but has some fluctuations.

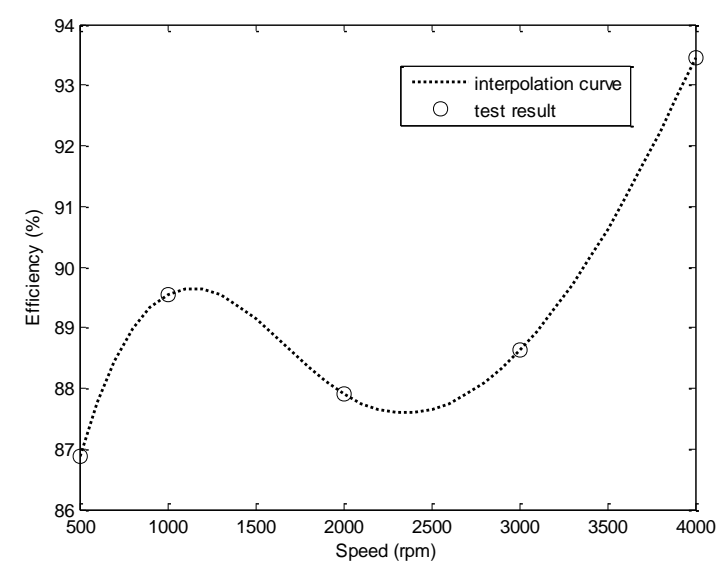

Figure8.Transmission efficiency with rotation rate at $5 \mathrm{Nm}$

\section{Conclusions}

Transmission efficiency is the key concerns in the design of gearing systems [10]. The results of analysis and experiment indicate that the energy storage devices exist in the system have important influence on the measurement of gear box's transmission. If the mathematical calculations only 
concern input and output power but not concern the intermediate links, the final test result may be larger than actual value. It is shown that the energy storage devices also can supply some energy as a power buffer. If there has flexible and/or elastic components and rotational inertia components in system, these interference factors must eliminate from the mathematical calculations to get the right test result.

\section{References}

1. T. Ciobotaru.D. Frunzeti.F. Jantschi. Method for analyzing epicyclic gearboxes. INT J AUTO TECH-KOR, 11, 2, 167-172(2010)

2. P. Alexandru, D. Macaveiu, C.Alexandru. A gear with translational wheel for a variable transmission ratio and applications to steering box. MECH MACH THEORY, 52, 267-276(2012)

3. S. Kirupasankar,C. Gurunathan,R.Gnanamoorthy, Transmission efficiency of polyamide nanocomposite spur gears. MATERIALS \& DESIGN, 39, 338-343(2012)

4. M. Mohammadpour,S.Theodossiades,H.Rahnejat,P.Kell.Transmission efficiency and noise, vibration and harshness refinement of differential hypoid gear pairs. Proceedings of the Institution of Mechanical Engineers, P I MECH ENG K-J MUL, 228, 1, 19-33( 2014)

5. Y.Iton, T. Sskaguchi. Development of the Mono Ring CVT - Principle and Mesurement of Transmission Efficiency. NTN TECHNICAL REVIEW,73,60-63(2005)

6. N.Chaudhari. Transmission Weight \& Efficiency Optimization in Off Road Vehicle (Tractor Gearbox). Control Theory and Informatics,4,1,58-61(2014)

7. A. M. Pande,Y. R. Kharde Effect of Pressure Angle on Transmission Efficiency of Helical Gears. IJITEE,3,2,42-45(2013)

8. Spicer,B. James.Effects of the Nonlinear Elastic Behavior of Bicycle Chain on Transmission Efficiency. J APPL MECH-T ASME,80, 2, ( 2013)

9. C. Qi, Z. Han, H. Kang.Study on calculation of transmission efficiency about the micro-segment gear. China Mechanical Engineering, 22, 13, 1537-9, (2011) Language: Chinese.

10. M.D.L. Cruz, S. Theodossiades, H. Rahnejat. An investigation of manual transmission drive rattle. Proceedings of the Institution of Mechanical Engineers, P I MECH ENG K-J MUL, 224, 2, 167-181( 2010) 